OPEN ACCESS

Edited by:

Hamad Ali,

Kuwait University, Kuwait

Reviewed by:

Asimina Mitrakou-Fanariotou,

National and Kapodistrian University of

Athens, Greece.

*Correspondence:

Xie-Hui Chen

xhchen66@126.com

Mei Qiu

13798214835@sina.cn

${ }^{t}$ ORCID:

Mei Qiu

orcid.org/0000-0001-5013-657X

Specialty section: This article was submitted to

Clinical Diabetes,

a section of the journal

Frontiers in Endocrinology

Received: 30 November 2021

Accepted: 15 December 2021

Published: 10 January 2022

Citation:

Zhao L-M, Chen X-H and Qiu M (2022) Commentary: Mortality Risk of

Antidiabetic Agents for Type 2

Diabetes With COVID-19: A

Systematic Review and Meta-Analysis.

Front. Endocrinol. 12:825100.

doi: 10.3389/fendo.2021.825100

\section{Commentary: Mortality Risk of Antidiabetic Agents for Type 2 Diabetes With COVID-19: A Systematic Review and Meta-Analysis}

\author{
Li-Min Zhao ${ }^{1}$, Xie-Hui Chen ${ }^{1 *}$ and Mei Qiu ${ }^{2 *}$ \\ ${ }^{1}$ Department of Geriatric Medicine, Shenzhen Longhua District Central Hospital, Shenzhen, China, ${ }^{2}$ Department of General \\ Medicine, Shenzhen Longhua District Central Hospital, Shenzhen, China
}

Keywords: COVID- 19, death, type 2 diabetes mellitus, SGLT2I, glp1 agonists, DPP4 (dipeptidyl peptidase 4) inhibitors

\section{A Commentary on}

Mortality Risk of Antidiabetic Agents for Type 2 Diabetes With COVID-19: A Systematic Review and Meta-Analysis

By Kan C, Zhang Y, Han F, Xu Q, Ye T, Hou N, et al. Mortality Risk of Antidiabetic Agents for Type 2 Diabetes With COVID-19: A Systematic Review and Meta-Analysis. Front Endocrinol (Lausanne) (2021) 12:708494. doi: 10.3389/fendo.2021.708494

\section{INTRODUCTION}

In a recent article (1), Kan and colleagues assessed the association between use of four kinds of hypoglycemic agents (i.e., metformin, sulfonylurea, dipeptidyl peptidase-4 inhibitors [DPP4i], and insulin) and risk of COVID-19 mortality in patients with type 2 diabetes mellitus (T2DM), by performing meta-analysis based on eligible studies. However, the authors did not address the association of two novel classes of hypoglycemic agents (i.e., sodium-glucose cotransporter-2 inhibitors [SGLT2i] and glucagon-like peptide-1 receptor agonists [GLP1RA]) with COVID-19 mortality, due to the limited number of included studies regarding SGLT2i and GLP1RA. Nowadays, more and more studies have been published, mainly targeting the relationship between SGLT2i or GLP1RA use and COVID-19 mortality risk (2-10). Therefore, we intended to include these recent studies regarding SGLT2i and GLP1RA to conduct an updated meta-analysis to address their association with COVID-19 mortality in T2DM patients. Moreover, several new cohort studies $(2-4,8,11-14)$ targeting the relationship of DPP4i use with COVID-19 death risk 
have been published after Kan et al.'s meta-analysis (1). Thus, in this updated meta-analysis we would also include these new DPP4i studies to reassess the association between use of DPP4i and risk of COVID-19 death in T2DM patients.

\section{METHODS}

Eligible studies for inclusion in this updated meta-analysis were cohort studies that enrolled T2DM patients with COVID-19 and estimated the COVID-19 mortality risk of use of DPP4i, SGLT2i, or GLP1RA, compared to no use of DPP4i, SGLT2i, or GLP1RA, respectively. The comparison of SGLT2i/GLP1RA use versus no SGLT2i/GLP1RA use included that of SGLT2i/GLP1RA use versus DPP4i use, whereas the comparison of DPP4i use versus no DPP4i use did not include that of DPP4i use versus SGLT2i/ GLP1RA use. Outcome of interest was COVID-19 death, and we preferred to use the most long-term outcome data. For example, if 7-day death (i.e., death within 7 days following hospital admission due to COVID-19) and 30-day death were both reported in included studies, we would use 30-day death. We searched PubMed, Cochrane Library, and Embase from the inception date of databases to November 21, 2021, using the main search terms: "COVID-19", "SARS-CoV-2", "type 2 diabetes mellitus", "DPP4i”, "SGLT2i", "GLP1RA", "death", and "mortality". Two authors independently performed study selection, quality assessment, and data extraction; and a third author addressed their divergences when necessary. The quality of included studies was assessed using the Newcastle-Ottawa Scale (15), according to which a quality score for included studies ranged from 0 to 9 , with a score of $\geq 7$ being regarded as "high quality".

We extracted the estimators of relative risk (RR) and 95\% confidence interval (CI) from included studies to perform metaanalysis. We preferentially extracted hazard ratio as RR, followed by risk ratio and odds ratio. Meta-analysis was conducted based on the comparisons of DPP4i versus Non-DPP4i, SGLT2i versus Non-SGLT2i, and GLP1RA versus Non-GLP1RA, respectively. Meta-analysis was done using a random-effects model when $\mathrm{I}^{2}$ $\geq 50 \%$, or using a fixed-effects model when $\mathrm{I}^{2}<50 \%$. We drew funnel plots and performed Egger's test to judge publication bias. Sensitivity analysis was conducted via the leave-one-out method (omitting one study each time), to evaluate the robustness of meta-analysis results. All statistical analyses were completed in Stata/MP 16.0.

\section{RESULTS}

We finally included 18 cohort studies $(2-14,16-20)$ in this metaanalysis. All of them were evaluated as "high quality". The detailed features of included studies and the outcome data extracted from them are presented in Table S1. Meta-analysis incorporating 16 studies (2-4, 7-14, 16-20) involving 549817 participants showed that compared to Non-DPP4i use, DPP4i use was significantly associated with a $17 \%$ reduction in COVID19 mortality risk (RR 0.83, 95\% CI 0.71-0.98, $\mathrm{P}=0.023$; Figure 1.1). Meta-analysis incorporating 6 studies (2-7) involving 275468 participants showed that compared to NonSGLT2i use, SGLT2i use was significantly associated with a $22 \%$ reduction in COVID-19 mortality risk (RR 0.78, 95\% CI 0.61$0.98, \mathrm{P}=0.035$; Figure 1.2). Meta-analysis incorporating 6 studies (2, 3, 6, 8-10) involving 140859 participants showed that compared to Non-GLP1RA use, GLP1RA use was significantly associated with a $25 \%$ reduction in COVID-19 mortality risk (RR 0.75, 95\% CI 0.60-0.94, P =0.013; Figure 1.3).

Publication bias was observed in the meta-analysis of DPP4i $\left(\mathrm{P}_{\text {Egger }}=0.012\right.$; Figure $\left.S 1\right)$, and was not observed in the metaanalyses of SGLT2i $\left(\mathrm{P}_{\text {Egger }}=0.885\right.$; Figure S2) and GLP1RA $\left(\mathrm{P}_{\text {Egger }}=0.092\right.$; Figure S3). The results of sensitivity analysis for DPP4i (Figure S4), SGLT2i (Figure S5), and GLP1RA (Figure S6) were similar with primary analysis results, which suggested the robustness of meta-analysis results.

\section{DISCUSSION}

In Kan and colleagues' article (1) the authors did not perform meta-analysis to assess the association between use of SGLT2i and GLP1RA and risk of COVID-19 mortality since they included only one SGLT2i study (7) and two GLP1RA studies $(9,10)$. On the contrary, we conducted meta-analysis respectively based on 6 SGLT2i studies (2-7) and 6 GLP1RA studies (2, 3, 6, $8-10)$, and accordingly revealed the significant association of SGLT2i use with a $22 \%$ reduction in COVID-19 mortality risk, and of GLP1RA use with a $25 \%$ reduction in that risk. Moreover, Kan et al.'s meta-analysis (1) did not include 8 recentlypublished DPP4i studies $(2-4,8,11-14)$, which limited its statistical power. Accordingly, a statistically significant difference between DPP4i and Non-DPP4i was not observed in that meta-analysis (1). In contrast, this updated meta-analysis included 16 DPP4i studies $(2-4,7-14,16-20)$ including the 8 recent ones $(2-4,8,11-14)$, and therefore with sufficient statistical power identified that DPP4i use versus Non-DPP4i use was significantly associated with a $17 \%$ reduction in COVID19 mortality risk.

One strength of this study is that sensitivity analyses suggested the robustness of meta-analysis results although possible publication bias was observed in DPP4i's metaanalysis. Oppositely, this study has two main weaknesses. First, we did meta-analyses based on cohort studies. Although we extracted the adjusted RRs and 95\% CIs from included studies to perform meta-analyses, there must be certain confounding factors unadjusted. Thus, relevant randomized trials are needed to verify our findings. Second, we failed to compare various hypoglycemic agents in COVID-19 death risk, and future studies comparing them are clinically meaningful. 


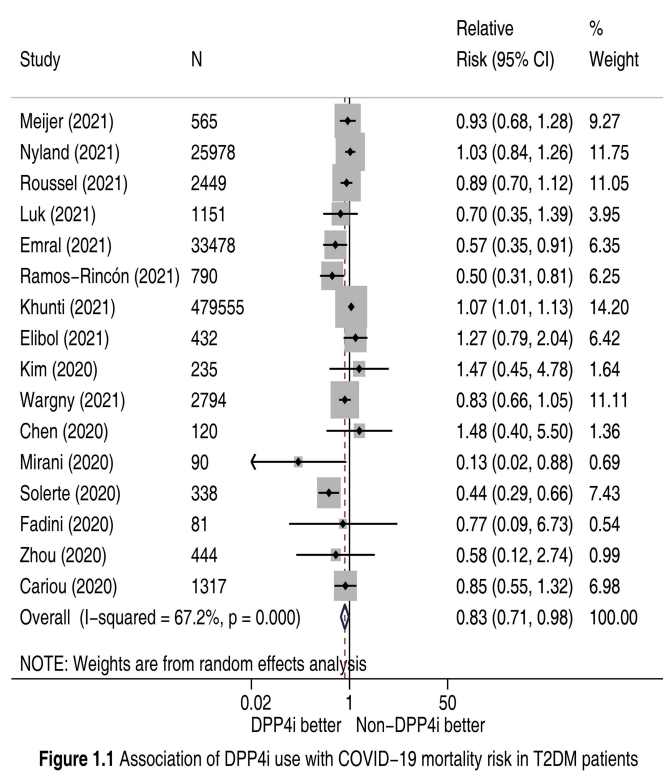

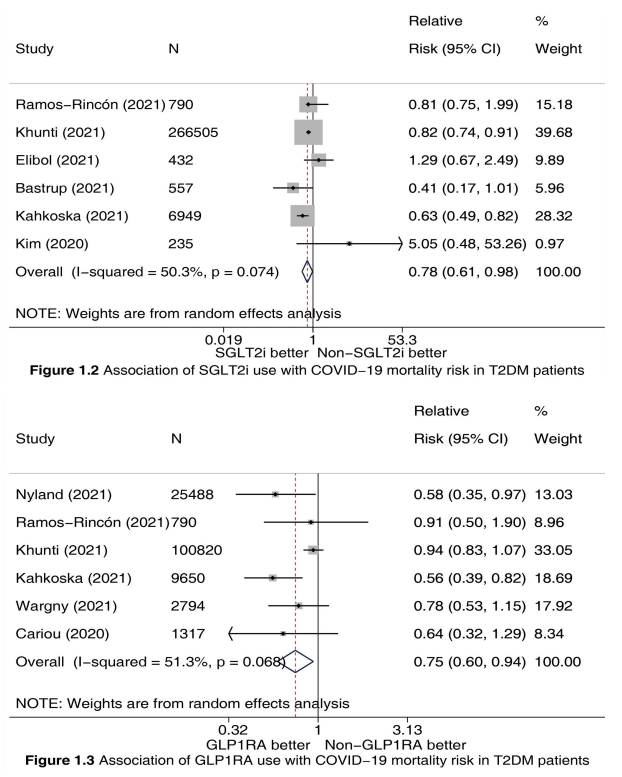

FIGURE 1 | Forest plots illustrating the association of DPP4i (Figure 1.1), SGLT2i (Figure 1.2), and GLP1RA (Figure 1.3) use with COVID-19 mortality risk in T2DM patients. DPP4i, dipeptidyl peptidase-4 inhibitors; SGLT2i, sodium-glucose cotransporter-2 inhibitors; GLP1RA, glucagon-like peptide-1 receptor agonists; T2DM, type 2 diabetes mellitus; $\mathrm{Cl}$, confidence interval.

Three new classes of hypoglycemic agents (i.e., SGLT2i, GLP1RA, and DPP4i) were significantly associated with reduced risk of COVID-19 death in T2DM patients with COVID-19, which highlights the potential of these new drugs used to prevent COVID-19 death in T2DM patients with COVID-19. Further studies are needed to assess whether there are significant differences in the risk of COVID-19 death among various kinds of hypoglycemic agents.

\section{AUTHOR CONTRIBUTIONS}

Design: MQ. Conduct/data collection: L-MZ, X-HC, and MQ. Analysis: L-MZ, and X-HC. Writing manuscript: L-MZ. Reviewing and editing manuscript: X-HC, and MQ. All authors contributed to the article and approved the submitted version.

\section{REFERENCES}

1. Kan C, Zhang Y, Han F, Xu Q, Ye T, Hou N, et al. Mortality Risk of Antidiabetic Agents for Type 2 Diabetes With COVID-19: A Systematic Review and Meta-Analysis. Front Endocrinol (Lausanne) (2021) 12:708494. doi: 10.3389/fendo.2021.708494

2. Ramos-Rincón JM, Pérez-Belmonte LM, Carrasco-Sánchez FJ, Jansen-Chaparro S, De-Sousa-Baena M, Bueno-Fonseca J, et al. Cardiometabolic Therapy and Mortality in Very Old Patients With Diabetes Hospitalized Due to COVID-19. J Gerontol A Biol Sci Med Sci (2021) 76(8):e102-9. doi: 10.1093/gerona/glab124

3. Khunti K, Knighton P, Zaccardi F, Bakhai C, Barron E, Holman N, et al. Prescription of Glucose-Lowering Therapies and Risk of COVID-19

\section{SUPPLEMENTARY MATERIAL}

The Supplementary Material for this article can be found online at: https:/www.frontiersin.org/articles/10.3389/fendo.2021.825100/ full\#supplementary-material

Supplementary Figure 1 | Test of publication bias for the meta-analysis of DPP4i versus Non-DPP4i.

Supplementary Figure 2 | Test of publication bias for the meta-analysis of SGLT2i versus Non-SGLT2i.

Supplementary Figure 3 | Test of publication bias for the meta-analysis of GLP1RA versus Non-GLP1RA.

Supplementary Figure 4 | Sensitivity analysis of DPP4i versus Non-DPP4i.

Supplementary Figure 5 | Sensitivity analysis of SGLT2i versus Non-SGLT2i.

Supplementary Figure 6 | Sensitivity analysis of GLP1RA versus Non-GLP1RA.

Supplementary Table 1 | Features of included studies and outcome data extracted from them.

Mortality in People With Type 2 Diabetes: A Nationwide Observational Study in England. Lancet Diabetes Endocrinol (2021) 9(5):293-303. doi: 10.1016/S2213-8587(21)00050-4

4. Elibol A, Eren D, Erdoğan MD, Elmaağaç M, Dizdar OS, Çelik O, et al. Factors Influencing on Development of COVID-19 Pneumonia and Association With Oral Anti-Diabetic Drugs in Hospitalized Patients With Diabetes Mellitus. Prim Care Diabetes (2021) 15(5):806-12. doi: 10.1016/j.pcd.2021.08.001

5. Israelsen SB, Pottegård A, Sandholdt H, Madsbad S, Thomsen RW, Benfield T. Comparable COVID-19 Outcomes With Current Use of GLP-1 Receptor Agonists, DPP-4 Inhibitors or SGLT-2 Inhibitors Among Patients With Diabetes Who Tested Positive for SARS-CoV-2. Diabetes Obes Metab (2021) 23(6):1397-401. doi: 10.1111/dom.14329 
6. Kahkoska AR, Abrahamsen TJ, Alexander GC, Bennett TD, Chute CG, Haendel MA, et al. Association Between Glucagon-Like Peptide 1 Receptor Agonist and Sodium-Glucose Cotransporter 2 Inhibitor Use and COVID-19 Outcomes. Diabetes Care (2021) 44(7):1564-72. doi: 10.2337/dc21-0065

7. Kim MK, Jeon JH, Kim SW, Moon JS, Cho NH, Han E, et al. The Clinical Characteristics and Outcomes of Patients With Moderate-To-Severe Coronavirus Disease 2019 Infection and Diabetes in Daegu, South Korea. Diabetes Metab J (2020) 44(4):602-13. doi: 10.4093/dmj.2020.0146

8. Nyland JE, Raja-Khan NT, Bettermann K, Haouzi PA, Leslie DL, Kraschnewski JL, et al. Diabetes, Drug Treatment and Mortality in COVID-19: A Multinational Retrospective Cohort Study. Diabetes (2021) 70:2903-16. doi: 10.2337/db21-0385

9. Wargny M, Potier L, Gourdy P, Pichelin M, Amadou C, Benhamou PY, et al. Predictors of Hospital Discharge and Mortality in Patients With Diabetes and COVID-19: Updated Results From the Nationwide CORONADO Study. Diabetologia (2021) 64(4):778-94. doi: 10.1007/s00125-020-05351-w

10. Cariou B, Hadjadj S, Wargny M, Pichelin M, Al-Salameh A, Allix I, et al. Phenotypic Characteristics and Prognosis of Inpatients With COVID-19 and Diabetes: The CORONADO Study. Diabetologia (2020) 63(8):1500-15. doi: 10.1007/s00125-020-05180-x

11. Meijer RI, Hoekstra T, van den Oever N, Simsek S, van den Bergh JP, Douma RA, et al. Treatment With a DPP-4 Inhibitor at Time of Hospital Admission for COVID-19 is Not Associated With Improved Clinical Outcomes: Data From the COVID-PREDICT Cohort Study in the Netherlands. J Diabetes Metab Disord (2021) 20:1-6. doi: 10.1007/s40200-021-00833-Z

12. Roussel R, Darmon P, Pichelin M, Goronflot T, Abouleka Y, Ait BL, et al. Use of Dipeptidyl Peptidase-4 Inhibitors and Prognosis of COVID-19 in Hospitalized Patients With Type 2 Diabetes: A Propensity Score Analysis From the CORONADO Study. Diabetes Obes Metab (2021) 23(5):1162-72. doi: 10.1111/dom.14324

13. Luk A, Yip T, Zhang X, Kong A, Wong VW, Ma R, et al. Glucose-Lowering Drugs and Outcome From COVID-19 Among Patients With Type 2 Diabetes Mellitus: A Population-Wide Analysis in Hong Kong. BMJ Open (2021) 11 (10):e52310. doi: 10.1136/bmjopen-2021-052310

14. Emral R, Haymana C, Demirci I, Tasci I, Sahin M, Cakal E, et al. Lower COVID19 Mortality in Patients With Type 2 Diabetes Mellitus Taking Dipeptidyl Peptidase-4 Inhibitors: Results From a Turkish Nationwide Study. Diabetes Ther (2021) 12(11):2857-70. doi: 10.1007/s13300-021-01133-8

15. Stang A. Critical Evaluation of the Newcastle-Ottawa Scale for the Assessment of the Quality of Nonrandomized Studies in Meta-Analyses. Eur J Epidemiol (2010) 25(9):603-5. doi: 10.1007/s10654-010-9491-z
16. Chen Y, Yang D, Cheng B, Chen J, Peng A, Yang C, et al. Clinical Characteristics and Outcomes of Patients With Diabetes and COVID-19 in Association With Glucose-Lowering Medication. Diabetes Care (2020) 43 (7):1399-407. doi: 10.2337/dc20-0660

17. Mirani M, Favacchio G, Carrone F, Betella N, Biamonte E, Morenghi E, et al. Impact of Comorbidities and Glycemia at Admission and Dipeptidyl Peptidase 4 Inhibitors in Patients With Type 2 Diabetes With COVID-19: A Case Series From an Academic Hospital in Lombardy, Italy. Diabetes Care (2020) 43(12):3042-9. doi: 10.2337/dc20-1340

18. Solerte SB, D’Addio F, Trevisan R, Lovati E, Rossi A, Pastore I, et al. Sitagliptin Treatment at the Time of Hospitalization was Associated With Reduced Mortality in Patients With Type 2 Diabetes and COVID-19: A Multicenter, Case-Control, Retrospective, Observational Study. Diabetes Care (2020) 43 (12):2999-3006. doi: 10.2337/dc20-1521

19. Fadini GP, Morieri ML, Longato E, Bonora BM, Pinelli S, Selmin E, et al. Exposure to Dipeptidyl-Peptidase-4 Inhibitors and COVID-19 Among People With Type 2 Diabetes: A Case-Control Study. Diabetes Obes Metab (2020) 22 (10):1946-50. doi: 10.1111/dom.14097

20. Zhou JH, Wu B, Wang WX, Lei F, Cheng X, Qin JJ, et al. No Significant Association Between Dipeptidyl Peptidase-4 Inhibitors and Adverse Outcomes of COVID-19. World J Clin Cases (2020) 8(22):5576-88. doi: $10.12998 /$ wjcc.v8.i22.5576

Conflict of Interest: The authors declare that the research was conducted in the absence of any commercial or financial relationships that could be construed as a potential conflict of interest.

Publisher's Note: All claims expressed in this article are solely those of the authors and do not necessarily represent those of their affiliated organizations, or those of the publisher, the editors and the reviewers. Any product that may be evaluated in this article, or claim that may be made by its manufacturer, is not guaranteed or endorsed by the publisher.

Copyright (c) 2022 Zhao, Chen and Qiu. This is an open-access article distributed under the terms of the Creative Commons Attribution License (CC BY). The use, distribution or reproduction in other forums is permitted, provided the original author(s) and the copyright owner(s) are credited and that the original publication in this journal is cited, in accordance with accepted academic practice. No use, distribution or reproduction is permitted which does not comply with these terms. 